\title{
Transient Deformation Regime in Bending of Single-Walled Carbon Nanotubes
}

\author{
A. Kutana and K. P. Giapis* \\ Division of Chemistry and Chemical Engineering, California Institute of Technology, Pasadena, California 91125, USA
}

(Received 14 August 2006; published 13 December 2006)

\begin{abstract}
Pure bending of single-walled carbon nanotubes between $(5,5)$ and $(50,50)$ is studied using molecular dynamics based on the reactive bond order potential. Unlike smaller nanotubes, bending of $(15,15)$ and larger ones exhibits an intermediate deformation in the transition between the buckled and fully kinked configurations. This transient bending regime is characterized by a gradual and controllable flattening of the nanotube cross section at the buckling site. Unbending of a kinked nanotube bypasses the transient bending regime, exhibiting a hysteresis due to van der Waals attraction between the tube walls at the kinked site.
\end{abstract}

DOI: 10.1103/PhysRevLett.97.245501

Carbon nanotubes possess remarkable mechanical properties [1-7], making them ideal candidates for nanoelectromechanical systems. Indeed, new nanodevices based on carbon nanotubes have been demonstrated, including a mass sensor [6], an oscillator [8], actuators [9], and a torsional pendulum [10]. A quantitative description of the elastic properties of nanotubes is necessary to understand their superior structural flexibility and expand their nanoscale applications.

Experimental [2,4,5] and theoretical [11-15] studies of basic loading types in carbon nanotubes have established that, when deformed, carbon nanotubes behave like elastic shells. This observation stimulated measurements and calculations of Young's modulus [3,7,16,17] and the Poisson's ratio [7] of nanotubes. Yacobson et al. [3] employed molecular dynamics (MD) simulations to study pure bending of small $(R \sim 5 \AA)$ nanotubes. Cao and Chen [12] estimated the critical buckling strain and curvature of larger nanotubes (with radii up to $27 \AA$ ) using both MD and finite elements. These studies identified a single critical discontinuity between bending and buckling of nanotubes, which was delineated by a change in the functional dependence of the strain energy of the nanotube on bending angle. The dependence was quadratic in the prebuckling regime but became linear beyond the buckling point, which was marked by the appearance of a distinct kink [2,3]. For small nanotubes, there is little difference between the just-buckled and kinked configurations. For example, Fig. 1(a) shows the shape of a $(10,10)$ single-walled carbon nanotube just after buckling. The nanotube walls at the buckling point are so close that further bending alters the nanotube cross section only slightly. However, this is not the case for larger diameter nanotubes. The just-buckled wall of a $(30,30)$ nanotube, shown in Fig. 1(b), is further from the opposite side. Therefore, more bending is required to bring the two sides close enough (i.e., to the equilibrium van der Waals distance) to form the kink.

In this Letter, we present results from MD simulations of large nanotubes $(R \geq 10 \AA)$, which predict the existence of an intermediate bending regime in the transition be-
PACS numbers: $62.25 .+\mathrm{g}, 02.70 . \mathrm{Ns}, 46.32 .+\mathrm{x}, 61.46 . \mathrm{Fg}$

tween the just-buckled and fully buckled (termed "kinked") configurations. This "transient bending regime" (TBR) is characterized by a gradual flattening of the nanotube cross section at the bending site until the nanotube walls collapse to form the kink. The strain energy curve exhibits two critical discontinuity points, clearly demarcating the TBR. Remarkably, unbending of a kinked nanotube does not retrace the TBR. As the bending angle is reduced, the tube walls remain collapsed until they abruptly spring up to the prebuckled state.

Molecular dynamics simulations of bending deformations in single-walled nanotubes (SWNTs) were performed using a code developed by Brenner et al., which implements a reactive bond order (REBO) potential for hydrocarbons [18]. The REBO potential has been shown to correctly reproduce the energy of various structural arrangements of carbon, including the 3-coordinated configuration in graphene tubules [19,20]. Significant van der Waals interactions exist between proximal nanotube walls as, for example, in the kinked configuration. Such nonbonding interactions were described with a pairwise 6-12 Lennard-Jones potential, with distance and energy parameters of $\sigma_{\mathrm{C}-\mathrm{C}}=3.407 \AA$ and $\varepsilon_{\mathrm{C}-\mathrm{C}} / k_{B}=$ $34.4 \mathrm{~K}$, respectively, for carbon-carbon atom pairs [21]. All simulations were quasistatic. Test runs at $300 \mathrm{~K}$ using a
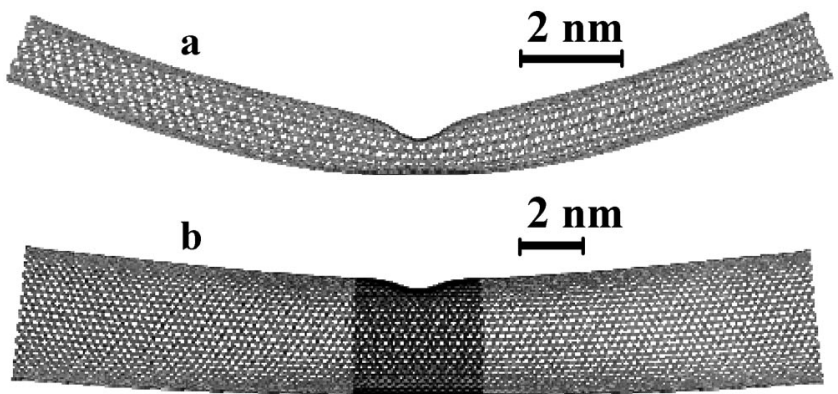

FIG. 1. Predicted shape of single-walled carbon nanotubes just after buckling, based on MD simulations. (a) 15.7-nm-long $(10,10)$ SWNT at $\theta=43^{\circ}$ and (b) 23.6-nm-long $(30,30)$ SWNT at $\theta=23^{\circ}$. Note the difference in scale. 
Berendsen thermostat indicated that the transition points between deformation regimes occurred at slightly smaller bending angles but did not otherwise affect the results.

The initial setup of a relaxed straight nanotube was prepared by minimizing the potential energy of the entire nanotube. During bending, the nanotube was divided into three segments: two fixed ends with a length of $\sim 5 \AA$, which were externally controlled and positioned to satisfy the boundary condition for the bending angle, and a central portion whose atoms were allowed to move.

The bending deformation was carried out by rotating the fixed ends in steps of $0.5^{\circ}$ around a line passing through the middle of the nanotube and normal to its center axis. After each step, the potential energy of the central segment was minimized using the conjugate gradient method. Additionally, the axial strain was removed through a succession of end displacements and central part relaxations. All nanotube lengths reported below refer to the extent of the central nanotube portion, prior to the onset of bending.

Previous simulations of buckling in carbon nanotubes have shown that the strain energy of the deformed nanotube varies nearly quadratically with the bending angle in the prebuckling regime and is close to linear in the kinking regime $[2,3,15]$. Our simulations of bending of $(5,5)$ and $(10,10)$ nanotubes agree well with elasticity theory calculations and reproduce results from previous MD studies for similar SWNTs [2-4]. Indeed, we find only one discontinuity in the potential energy of these nanotubes as they transition from the prebuckled to the kinked configuration. However, as the diameter of the nanotube is increased, a second discontinuity appears in the potential energy curve at a larger bending angle than the first one. This behavior is illustrated in Fig. 2, where the strain energy $U$ and the bending moment $M=d U / d \theta$ are plotted as a function of bending angle $\theta$ for a $(30,30)$ SWNT. Three distinct deformation regimes are observed for this nanotube, clearly separated by discontinuity points at $\theta=12^{\circ}$ and $32^{\circ}$ [22]. It is instructive to discuss these regimes in view of the corresponding nanotube longitudinal shape and cross section at the buckling point, shown in Fig. 3. In the initial elastic regime, the strain energy $U$ exhibits a quadratic dependence on the bending angle, while the cross section experiences progressive ovalization as the bending angle increases, culminating to the shape in Fig. 3(a). The buckling event is marked by an abrupt transition from the oval cross section to one with the flat top shown in Fig. 3(b). The bending moment's rate of growth switches from positive before buckling to negative after buckling.

As the bending angle increases during the TBR, the flat portion of the top wall expands continuously across the nanotube [see Figs. 3(c) and 3(d)], while the bottom wall retains its oval shape, albeit with less curvature. As a result, the top-to-bottom wall distance decreases gradually, reducing the tube cross section at the buckling site. The strain energy dependence on the bending angle is no longer

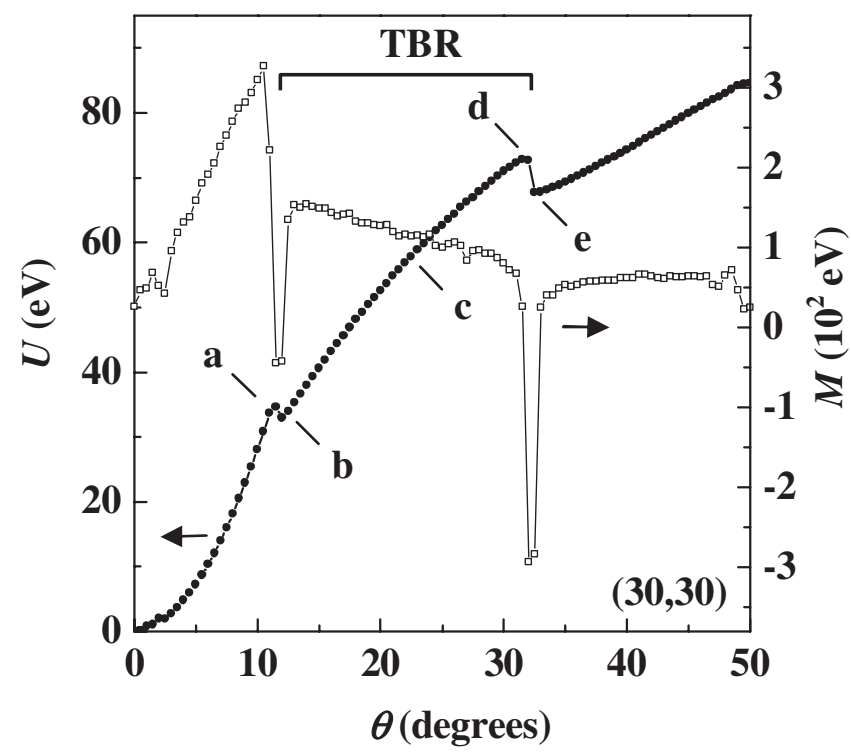

FIG. 2. Strain energy $U$ and bending moment $M=d U / d \theta$ for a 23.6-nm-long $(30,30)$ SWNT as a function of the bending angle $\theta$. The letters a-e indicate the points for which the tube shape and cross section at the buckling point are shown in Fig. 3. TBR denotes the transient bending regime.

quadratic - in fact, the exponent becomes less than 1 . When the approaching opposite walls reach the van der Waals equilibrium distance of $3.4 \AA$ [Fig. 3(e)], the cross section collapses, forming the kink, and a second discontinuity is observed. The third bending regime begins at this point, with no significant change in the shape of the cross section upon further bending. This regime is characterized by a relatively constant bending moment.

Three deformation regimes are observed for all SWNTs with diameters between $(15,15)$ and the largest studied $(50,50)$. Nanotube size determines the range of bending angles over which the TBR occurs, as well as the magnitude of the energy drop when the tube cross section collapses. Interestingly, the TBR is fully reversible: If bending is stopped before the second discontinuity occurs, unbending recovers the cross-sectional shapes at the buckling point. The reversible variation in the area of the cross section during the TBR may act as a means of restricting the flow of a liquid in the inner cavity of a SWNT and thus serve as a nanoconduit valve.

Unlike linear elastic bending, the deformation of nanotubes during the TBR is inhomogeneous: The strain energy is not constant along the tube length. It is instructive to consider the energetics of the buckled cross section and the nanotube end portions separately. Figure 4 compares the potential energy of a $(30,30)$ tube segment $(\sim 1 / 3$ of the tube length) away from the buckling site to that of a thin ring consisting of 300 carbon atoms at the buckling site. The onset of buckling corresponds to a redistribution of strain: The outer portions relax, while the ring at the buckling site gains a substantial amount of strain energy. 


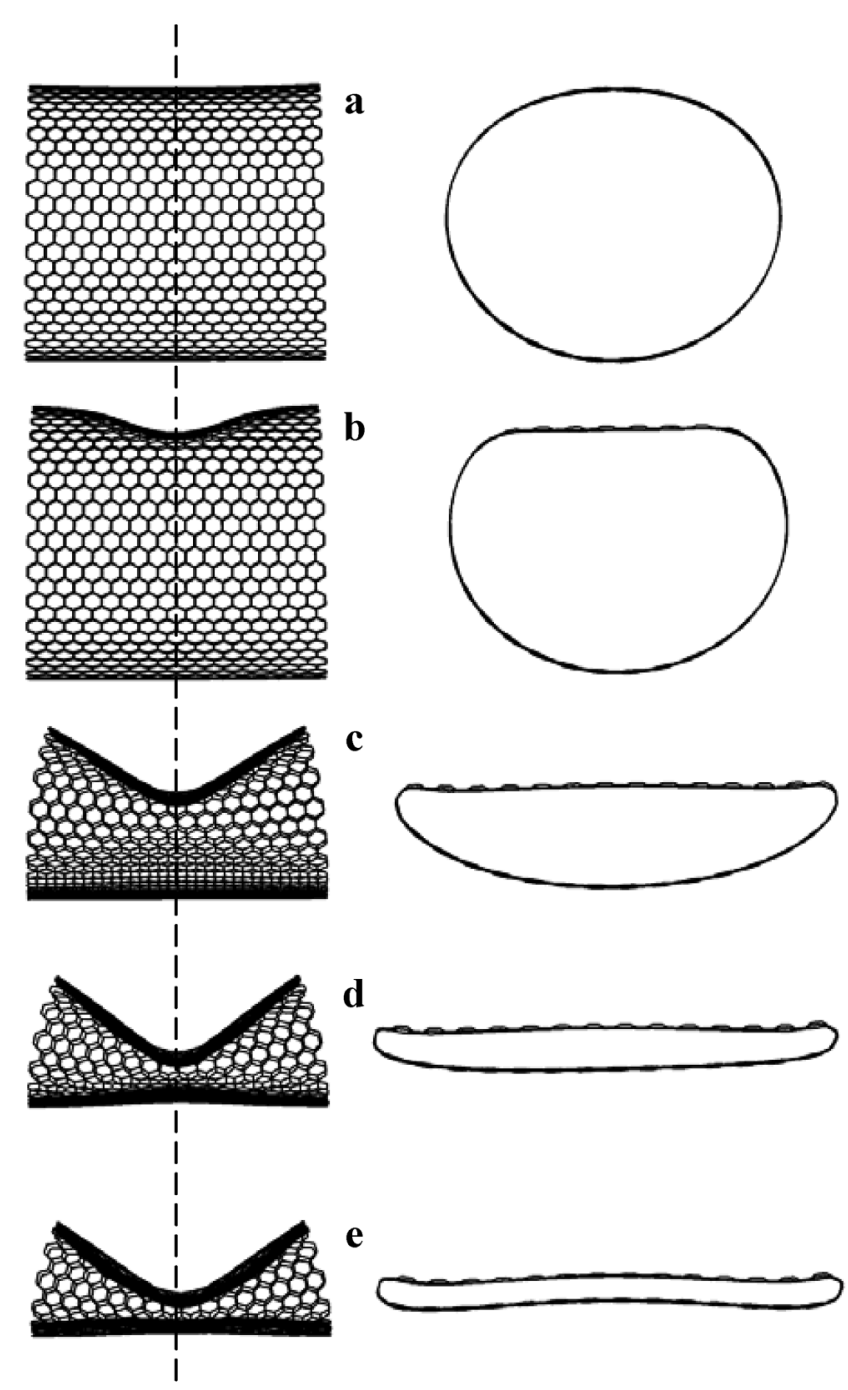

FIG. 3. Side views and buckling point cross sections of a 23.6nm-long $(30,30)$ SWNT at various bending angles corresponding to the points a-e marked on the strain energy curve in Fig. 2. Only the portion near the buckling region [darkened region in Fig. 1(b)] is shown for the longitudinal views. Bending occurs in the plane of the journal page.

In comparison, the energy gain of an equal-sized ring near the nanotube end is insignificant, as shown in Fig. 4. Upon further bending, the strain energy of the end segment decreases slightly, while that of the buckling region continues to increase significantly. This behavior may be qualitatively explained by considering the tube as an elastic shell. It is well known from the theory of elasticity that the cross section of a uniformly bent cylindrical shell is ovalized by a radial force, which ensues from the variation in the direction of the tensile stress [23,24]. A much greater compressive force is applied to the buckling area cross section during the TBR, because the change in the stress direction is more abrupt, causing the progressive collapse of the cross section. The nanotube ends may be considered as effective levers that amplify the force exerted on the
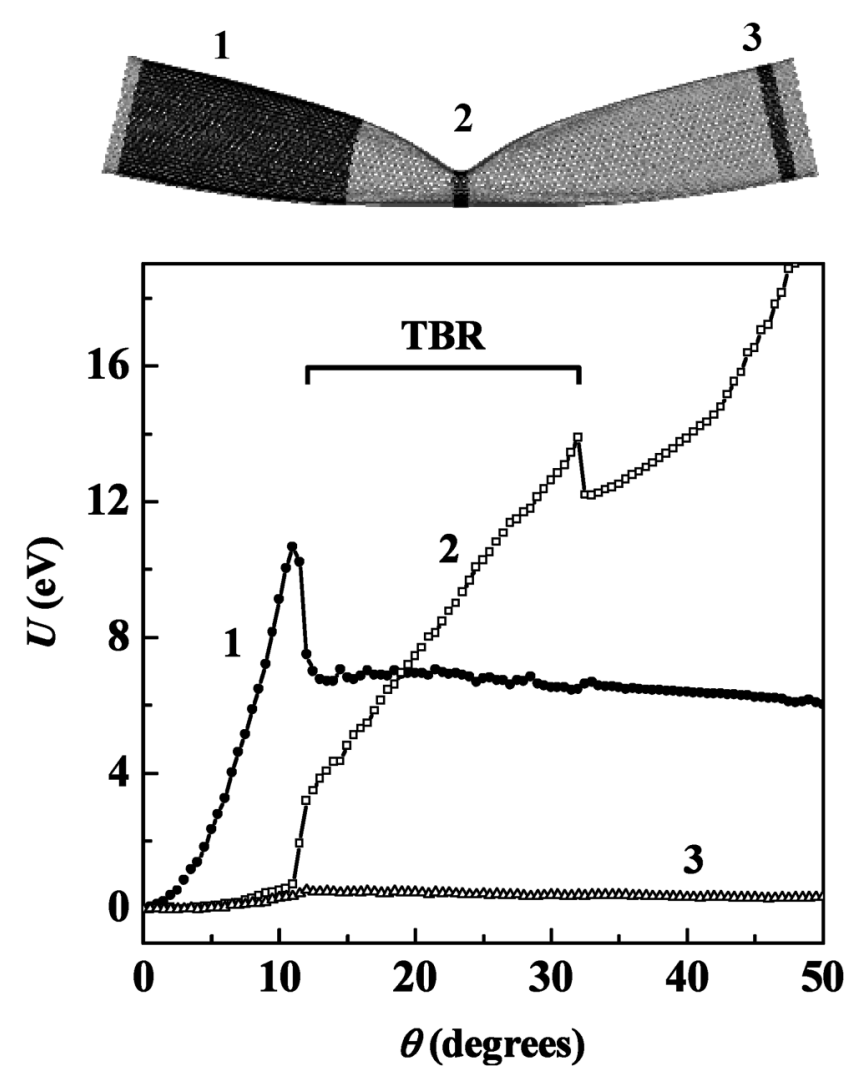

FIG. 4. Strain energy of various segments of a 23.6-nm-long $(30,30)$ SWNT as a function of the bending angle $\theta$. (1) Tube segment $(\sim 1 / 3$ of SWNT length) away from buckling region, (2) thin ring at the buckling point, and (3) thin ring away from buckling point. The van der Waals energy is excluded. The TBR region is observed between $12^{\circ}$ and $32^{\circ}$.

compressed cross section at the buckling site. van der Waals interactions are of no significance until the second critical discontinuity.

The kinked configuration is marked by the collapse of the nanotube walls at the buckling site. MD simulations of SWNTs have predicted that the strain energies of the circular and collapsed configurations are equal at $R_{\mathrm{SWNT}} \simeq$ $R_{0}=31.2 \AA$ [25]. When $12.1 \AA<R_{\mathrm{SWNT}}<R_{0}$, the collapsed configuration is metastable, and the cylindrical configuration is more stable, while the opposite is true for $R_{\mathrm{SWNT}}>R_{0}$. Our simulations predict that bistable switching between the collapsed and cylindrical forms can be observed at the kinking site in SWNTs whose radius is slightly less than $R_{0}$. Figure 5(a) compares the strain energy of bending and unbending of a $(30,30)$ nanotube $(R=20.3 \AA$ ). Remarkably, unbending after the occurrence of the second discontinuity point bypasses entirely the intermediate bending regime. Rather, the nanotube switches directly from the kinking to the linear elastic regime. The kink is preserved by attractive van der Waals forces between the collapsed nanotube walls, which are responsible for the unbending hysteresis. 


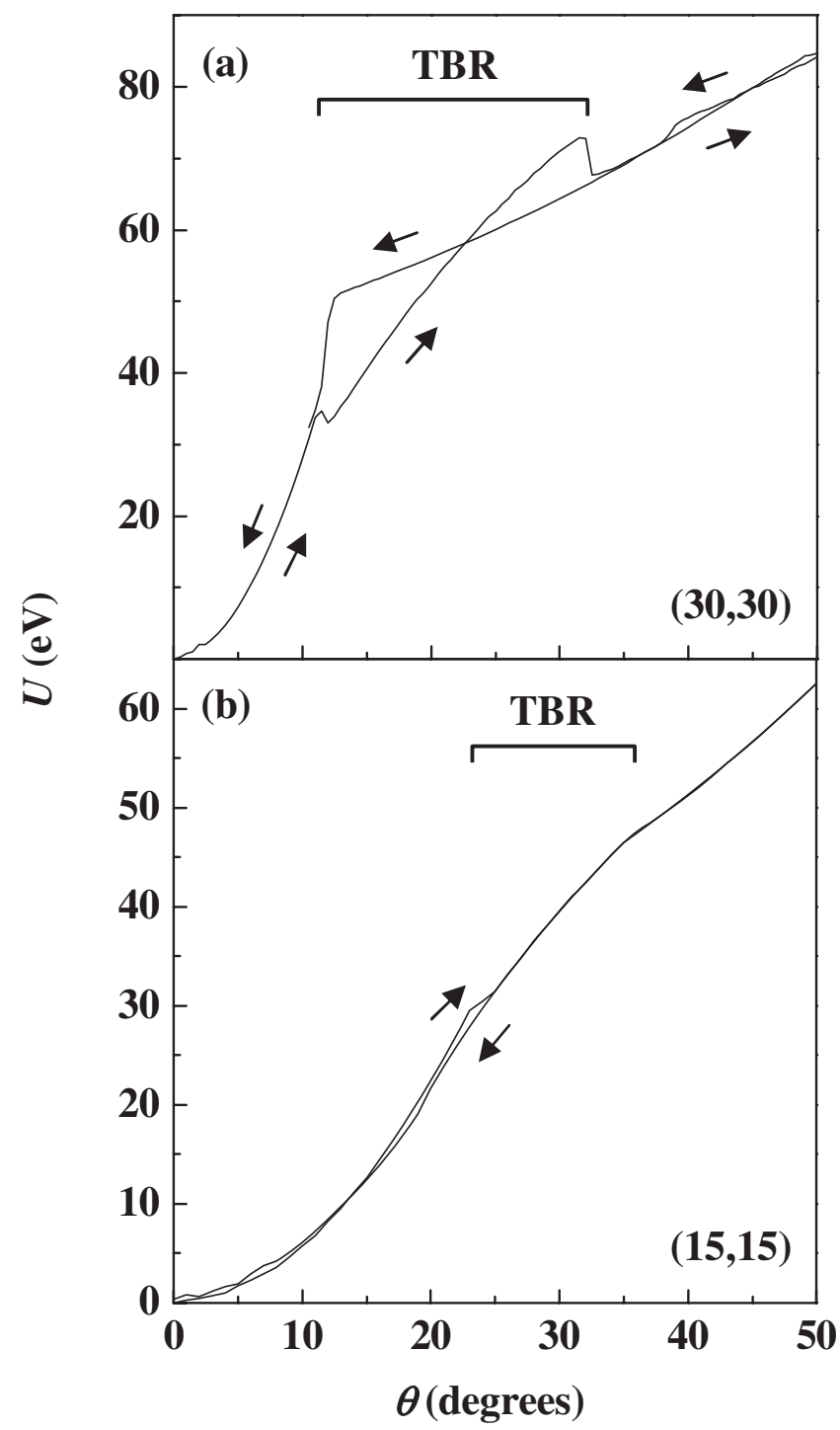

FIG. 5. (a) Strain energy $U$ for a 23.6-nm-long (30, 30) SWNT during bending and unbending operations. While all three bending regimes are present during bending, unbending proceeds by a direct switch from the kinking to the linear elastic regime. (b) Strain energy of bending and unbending of a $(15,15)$ SWNT. The energy does not depend on the direction of bending. TBR denotes the transient bending regime.

In smaller nanotubes, the mutual wall attraction is not sufficient to produce enough cohesion for the unbending hysteresis to be observed. For instance, Fig. 5(b) shows the energy of bending and unbending of a 15.7-nm-long $(15,15)$ SWNT, where no dependence on the direction of bending is found. Unbending recovers the geometrical shapes observed during bending, including those seen in the intermediate TBR. This is also true for the $(30,30)$ SWNT, when van der Waals forces are neglected (not shown). In that case, unbending retraces the bending pathway through the transient bending regime. Thus, we con- clude that van der Waals interactions play a crucial role in the manifestation of the unbending hysteresis in nanotubes.

In conclusion, MD simulations of pure bending of SWNTs reveal that nanotubes with size $(15,15)$ and up exhibit a transient bending regime, between the linear elastic and nonlinear kinking regimes expected for smaller nanotubes. This intermediate regime is characterized by a gradual flattening of the compressed side of the nanotube and a concomitant reduction of the cross section at the bending site. Unbending of fully kinked nanotubes bypasses the transient bending regime, exhibiting a hysteresis due to van der Waals attraction between the collapsed walls at the kinked site.

This work was based on research supported by NSF (No. CTS-0508096).

*Corresponding author. Electronic address: giapis@cheme.caltech.edu

[1] M. M. J. Treacy, T. W. Ebbesen, and J. M. Gibson, Nature (London) 381, 678 (1996).

[2] S. Iijima et al., J. Chem. Phys. 104, 2089 (1996).

[3] B. I. Yakobson, C. J. Brabec, and J. Bernholc, Phys. Rev. Lett. 76, 2511 (1996).

[4] E. W. Wong, P. E. Sheehan, and C. M. Lieber, Science 277, 1971 (1997).

[5] M. R. Falvo et al., Nature (London) 389, 582 (1997).

[6] P. Poncharal et al., Science 283, 1513 (1999).

[7] D. Sanchez-Portal et al., Phys. Rev. B 59, 12678 (1999).

[8] V. Sazonova et al., Nature (London) 431, 284 (2004).

[9] R. H. Baughman et al., Science 284, 1340 (1999).

[10] J.C. Meyer, M. Paillet, and S. Roth, Science 309, 1539 (2005).

[11] T. Ozaki, Y. Iwasa, and T. Mitani, Phys. Rev. Lett. 84, $1712(2000)$

[12] G. Cao and Xi Chen, Phys. Rev. B 73, 155435 (2006).

[13] M. Arroyo and T. Belytschko, Phys. Rev. Lett. 91, 215505 (2003).

[14] A. Pantano, M.C. Boyce, and D. M. Parks, Phys. Rev. Lett. 91, 145504 (2003).

[15] T. Vodenitcharova and L. C. Zhang, Phys. Rev. B 69, 115410 (2004).

[16] A. Krishnan et al., Phys. Rev. B 58, 14013 (1998).

[17] J. P. Lu, Phys. Rev. Lett. 79, 1297 (1997).

[18] D. W. Brenner et al., J. Phys. Condens. Matter 14, 783 (2002).

[19] J. A. Elliott et al., Phys. Rev. Lett. 92, 095501 (2004).

[20] H. Liang and M. Upmanyu, Phys. Rev. Lett. 96, 165501 (2006).

[21] J.P. Lu, X.-P. Li, and R. M. Martin, Phys. Rev. Lett. 68, 1551 (1992).

[22] The exact bending angles at which the discontinuities appear depend on the size of the nanotube and also on temperature.

[23] L. G. Brazier, Proc. R. Soc. A 116, 104 (1927).

[24] C. R. Calladine, Theory of Shell Structures (Cambridge University Press, Cambridge, England, 1983).

[25] S. Zhang et al., Phys. Rev. B 73, 075423 (2006). 